\title{
Consequences of recruitment decisions and heterogeneity on age-specific breeding success in a long-lived seabird
}

\author{
Lise M. Aubry,${ }^{1,2,3,5}$ David N. Koons,${ }^{3}$ Jean-Yves Monnat, ${ }^{4}$ and Emmanuelle Cam ${ }^{2}$ \\ ${ }^{1}$ Max Planck Institute for Demographic Research, Konrad-Zuse Str. 1, D-18057 Rostock, Germany \\ ${ }^{2}$ Laboratoire Evolution et Diversité Biologique, Bâtiment IVR3 118 Route de Narbonne, 31062 Toulouse Cedex 09, \\ Université Paul Sabatier, France \\ ${ }^{3}$ Department of Wildland Resources and the Ecology Center, 5230 Old Main Hill, Utah State University, \\ Logan, Utah 84322-5230 USA \\ ${ }^{4}$ Penn ar Run Izella, Goulien, Cap Sizun, France
}

\begin{abstract}
An individual's age at first reproduction and investment in successive reproductive attempts are involved in mechanisms that can impede somatic repair, resulting in a decline in reproductive abilities with age (reproductive senescence). We used long-term data from the Black-legged Kittiwake, a long-lived seabird, to address the relationship between recruitment age, age-specific breeding success (BS), and reproductive senescence, while accounting for breeding experience and temporal variation in BS. We first detected late-life improvement in BS across all recruitment groups, which we recognized as "within-generation selection" or the selective disappearance of "frail" phenotypes. When such heterogeneity was accurately accounted for, we showed that all individuals suffered reproductive senescence. We first highlighted how different combinations of pre- and post-recruitment experience across recruitment groups resulted in maximal BS at intermediate ages. BS increased in early recruits as they gained post-recruitment experience, whereas late recruits gained pre-recruitment experience that led to high BS at recruitment. Only individuals recruiting at intermediate ages balanced their pre- and post-recruitment experience. Consistent with the "cumulative reproductive cost hypothesis," we also observed a faster decline in BS in early recruits at advanced ages, whereas individuals delaying recruitment experienced the slowest decline in BS with age. Early recruits, however, reached the highest levels of BS at intermediate ages, sensus stricto (10-13 years old), whereas individuals delaying recruitment experienced the lowest at similar ages. These divergent trajectories may reflect a "delayed trade-off" balancing a maximization of midlife BS against reproductive senescence at advanced ages. Additionally, annual variation in BS had a greater effect on individuals early in life, suggesting that experienced individuals were able to buffer out the effects of temporal variation on BS, which can ultimately improve fitness in stochastic environments. Our findings stress that (1) both observed and unobserved heterogeneity are important in detecting within-individual senescence, and (2) short-term trade-offs may be rare in long-lived species; thus, cumulated reproductive costs should be invoked as an alternative mechanism underlying reproductive senescence.
\end{abstract}

Key words: age-specific recruitment; Black-legged Kittiwake; breeding success; delayed cost of reproduction; heterogeneity; reproductive senescence; Rissa tridactyla; temporal variation; trade-offs.

\section{INTRODUCTION}

Within phylogenetic and environmental boundaries, long-lived organisms have to make a series of decisions such as when to breed for the first time, how many times to reproduce, and how much and when to devote limited resources away from reproduction toward maintenance or growth (i.e., life history trade-offs). The most widely studied trade-off is the cost of reproduction (for a review

Manuscript received 7 August 2008; revised 22 December 2009; accepted 8 January 2009. Corresponding Editor: J. P. Y. Arnould.

${ }^{5}$ Present address: Department of Wildland Resources, 5230 Old Main Hill, Utah State University, Logan, Utah 84322-5230 USA. E-mail: lise.aubry@aggiemail.usu.edu see Harshman and Zera 2007), in which investment in current reproductive effort is expected to decrease subsequent survival and (or) reproduction. However, empirical support for costs of reproduction in wild organisms remains ambiguous (Harshman and Zera 2007). For example, Cam (1997) and Cam et al. (1998, 2002a) observed a positive phenotypic correlation between survival and breeding probability in Blacklegged Kittiwakes (Rissa tridactyla; a long-lived seabird; see Plate 1). Individuals that bred successfully in a given year also survived and reproduced with a higher probability in the following year compared to failed breeders, and nonbreeders (individuals that skipped reproduction in a given year). In the latter case, individuals actually experienced a "cost of nonrepro- 
duction," as failure to breed in year $t$ was associated with lower breeding probability in year $t+1$ and lower chances of surviving up to $t+1$.

Positive relationships between fitness components are common in observational studies of long-lived species (e.g., Barbraud and Weimerskirch 2005, Nevoux et al. 2007). Three nonexclusive hypotheses may explain positive relationships between fitness components. First, heterogeneity among individuals in their ability to acquire resources may mask trade-offs (van Noordwijk and de Jong 1986). For this reason, it has been argued that trade-offs may not be detectable without using an experimental approach (Partridge and Barton 1983). Second, it has been hypothesized that trade-offs may be expressed only in poor environmental conditions, when resources are scarce (Stearns 1992). Last, the consequences of investing resources into reproduction may not be detectable immediately, and individuals may incur delayed costs (i.e., long-term costs on a lifetime scale).

If costs of reproduction are not demographically evident in the short term, payment could reveal itself later in life, even at advanced ages. For example, an "invest now, pay later" reproductive tactic could culminate in delayed or cumulative costs of reproduction (e.g., Orell and Belda 2002), and could translate into late-life senescence in reproduction. Nevertheless, the literature is heavily weighted toward examination of short-term reproductive costs (Harshman and Zera 2007), for two main reasons. First, adverse conditions and high levels of extrinsic mortality do not allow the large majority of individuals in wild populations to reach senescent ages because most die as juveniles or as young adults (e.g., Ricklefs and Sheuerlein 2001). Second, studies monitoring populations over a period of time long enough for senescence to be detected have come to fruition only in recent years; examples include mammals (Nussey et al. 2007), birds (Cam et al. 2002a, Charmantier et al. 2006), and fish (Reznick et al. 2004).

Quantifying delayed costs of reproduction would clarify the simultaneous evolution of delayed reproduction and senescence (Lack 1968). Age at first reproduction (i.e., recruitment timing) is often assumed to initiate mechanisms that impede somatic repair, resulting in a decline in reproductive abilities with age, i.e., reproductive senescence (e.g., Charnov 1997). If so, different recruitment tactics, such as early vs. delayed recruitment, could lead to contrasting reproductive trajectories (e.g., senescence or improvement in reproductive success with age).

A further complication is that reproductive experience gained throughout life can also have a strong influence on age-specific breeding success (i.e., BS). Increased breeding experience can even lead to improved BS with age (e.g., Nol and Smith 1987, Orell and Belda 2002), obscuring the influence of recruitment timing on reproductive senescence in wild populations. Variable environmental conditions can further affect BS and survival, and must be statistically controlled for in order to detect delayed costs of reproduction in the wild.

Here, we use data from a study of the Black-legged Kittiwake in which age at recruitment and age-specific BS have been recorded for $>2100$ individuals over almost 30 years. We address the relationship between recruitment decisions and reproductive senescence, while taking into account opportunities for breeding experience and temporal variation in reproductive conditions to improve or diminish BS across ages. Specifically, we propose two contrasting hypotheses.

1) The cumulative cost hypothesis is that early recruitment is associated with acute reproductive senescence (i.e., faster decline in BS at advanced ages), especially for the longest lived individuals (i.e., those that cumulate a greater number of breeding attempts over life). Recruits delaying first reproduction, on the other hand, are expected to incur a smaller cumulative reproductive cost, especially if short lived (i.e., delayed recruitment associated to a short reproductive life span translates into a low cost of cumulative breeding events over life). Thus, we expect senescence in reproduction for these birds to be subtle or absent.

2) The heterogeneity in individual quality hypothesis leads to opposite predictions: individuals of high intrinsic quality recruit early (e.g., Nur 1988) and experience high levels of BS late in life. This hypothesis assumes that higher quality individuals incur smaller reproductive costs than others for equal reproductive investment. Under this view, we may expect early recruits to incur smaller cumulative costs than others as they age and breed, and senescence to be absent or weak. In contrast, individuals of poor intrinsic quality delay recruitment and their senescent decline in reproduction is expected to be sharper. For an equal number of breeding events, greater cumulative costs of reproduction in low-quality individuals are expected to translate into a sharper decline in reproductive ability than in high-quality individuals.

These two hypotheses concerning senescence can be combined with a third: lack of pre-breeding experience may translate into poor BS at the beginning of reproductive life in early recruits. Thus, we may expect an initial increase in BS with experience. In contrast, late recruits may exhibit high $\mathrm{BS}$ in the year of recruitment and early in reproductive life because they had time to acquire the skills necessary for reproduction (e.g., mate coordination, knowledge of foraging places), and to "prospect" for higher quality sites. Prospectors are assumed to gather local information on their conspecific BS before settling and breeding for the first time (e.g., Danchin et al. 1991, 1998, Cadiou et al. 1994), which, if habitat quality is constant from one year to the next, allows identification of higher quality habitat, and thus may ensure high BS (e.g., Boulinier and Danchin 1997).

Coulson and Fairweather (2001) also studied late-life reproductive performance in Black-legged Kittiwakes. They observed a sharp decline in BS at the last breeding 
TABLE 1. Selection results for models testing the effects of age at first reproduction and experience on age-specific breeding success in the Black-legged Kittiwake (Rissa trudactyla).

\begin{tabular}{|c|c|c|c|c|c|}
\hline Model & $\mathrm{AIC}$ & edf & $\Delta_{i}$ & $\exp \left(-0.5 \Delta_{i}\right)$ & $w_{i}$ \\
\hline $\mathrm{S}(\mathrm{AFRc} \times \mathrm{EXP})$ & 10969 & 18 & 0 & 1 & 0.96 \\
\hline $\mathrm{AFRc} \times\left(\mathrm{EXP}+\mathrm{EXP}^{2}+\mathrm{EXP}^{3}\right)$ & 10976 & 20 & 6 & 0.05 & 0.04 \\
\hline $\mathrm{AFRc} \times\left(\mathrm{EXP}+\mathrm{EXP}^{2}\right)$ & 10984 & 15 & 14 & 0 & 0 \\
\hline $\mathrm{AFRc}+\mathrm{EXP}+\mathrm{EXP}^{2}+\mathrm{EXP}^{3}$ & 11057 & 8 & 87 & 0 & 0 \\
\hline $\mathrm{AFRc}+\mathrm{EXP}+\mathrm{EXP}^{2}$ & 11061 & 7 & 91 & 0 & 0 \\
\hline AFRc $\times$ EXP & 11068 & 10 & 98 & 0 & 0 \\
\hline $\mathrm{EXP}+\mathrm{EXP}^{2}+\mathrm{EXP}^{3}$ & 11093 & 4 & 123 & 0 & 0 \\
\hline $\mathrm{EXP}+\mathrm{EXP}^{2}$ & 11097 & 3 & 127 & 0 & 0 \\
\hline $\mathrm{AFRc}+\mathrm{EXP}$ & 11111 & 6 & 141 & 0 & 0 \\
\hline EXP & 11149 & 2 & 179 & 0 & 0 \\
\hline AFRc & 11297 & 5 & 327 & 0 & 0 \\
\hline
\end{tabular}

Notes: The top model is a GAM as it involves a spline function "S"; all other models are GLMs. Abbreviations: age at first reproduction (AFR if continuous, AFRc if categorical); experience (EXP if continuous, EXP ${ }^{2}$ if quadratic, EXP ${ }^{3}$ if cubic); " + " denotes an additive effect, " $X$ " an interaction; edf, estimated degrees of freedom; Akaike's information criterion AIC $=-2 \times$ $\log$-likelihood $+2 \times$ edf; $\Delta_{i}=\mathrm{AIC}_{\text {model } i}-\mathrm{AIC}_{\text {min }} ; w_{i}=\exp \left(-0.5 \Delta_{i}\right) / \Sigma_{i} \exp \left(-0.5 \Delta_{i}\right)$.

event, but did not otherwise find any evidence for senescence. Even though they asserted that "terminal illness" was independent of age and not a reflection of senescence, they did not study the entire breeding history and did not properly account for heterogeneity in individual quality, suggesting that their findings might not be as robust as previously thought. Here, our ultimate goal is to disentangle trade-offs between earlylife decisions and late-life BS from heterogeneity in individual quality, in order to identify the demographic mechanisms shaping age-specific breeding trajectories and reproductive senescence.

\section{Methods}

Information on the study population, data collection, sample specifications, crude data on BS, and sample size, is given in Appendix A.

\section{Age-related change in breeding success}

In this study, only individuals that survived until recruitment, and subsequently recruited, were considered in analyses of BS (2124 individuals, 8335 observations). By working on the sample of individuals that recruited, we could use generalized linear models, GLM (Agresti 1990); generalized additive models, GAM (Hastie and Tibshirani 1986); and generalized additive mixed models, GAMM (Wood 2006) to estimate the affect of age-related traits on BS from recruitment thereon.

To address the influence of age at first reproduction (AFR) and breeding experience (EXP, the time in years elapsed since first reproduction) on the probability of breeding successfully (BS), we treated BS as a binary response variable (i.e., success or failure) and first used GLM (i.e., logistic regression; see Agresti [1990]; package "MASS," procedure "glm" in R 2.6.0).

We used the term "experience" in the sense of an opportunity to increase breeding abilities through learning. In this population, experience largely captures the number of breeding attempts over life. Even sabbaticals (i.e., individuals that skipped breeding in a given year and make up only $10 \%$ of all breeding events) have the opportunity to gain experience by interacting with other individuals (e.g., Danchin et al. 1998), learning about foraging opportunities, and about hotspots (i.e., cliff locations where breeding pairs are producing viable offspring). Sabbaticals can thus learn about the quality of breeding sites in which they may try to breed in the future. Sabbaticals were included in the analyses, because we consider them to be part of the population "at risk"; they are able and susceptible to breed, but do not, and can therefore be considered as "failed breeders." Thus, the covariate referring to experience is a reasonable reflection of the number of breeding attempts over the life course.

We considered models with linear, quadratic, and cubic effects of EXP on BS (see Table 1). A quadratic effect translates into minimal (or maximal for negative coefficients) BS for individuals of intermediate experience, whereas a cubic relationship can account for a possible bimodal pattern in BS as a function of EXP. We considered EXP rather than age to investigate the idea of cumulative costs of reproduction, where (along with AFR) only post-reproductive experience matters, and not age sensus stricto. We also considered AFR as either a categorical or continuous covariate in our analysis. When AFR was treated as a categorical covariate, we conducted a cluster analysis (package "stats," procedure "hclust" in R version 2.6.0; R Development Core Team 2007) to choose our cutting points in order to ensure that the selected recruitment groups were statistically meaningful. When performing a cluster analysis, we used Ward's test, which generates clusters minimizing the squared Euclidean distance to the center mean. According to this method, our recruitment groups were defined as: recruitment at age 3, 4, 5, 6, and 7 years or older (7+).

Because GLMs with continuous covariates assume a specific shape (e.g., linear, quadratic) for the relationship between explanatory and response variables, we compared the fit of our best GLM to the fit of a less constrained GAM (package "mgcv," procedure "gam" 
in $\mathrm{R}$ version 2.6.0). The generalized additive model (GAM) alleviates constraints of the GLM by fitting nonparametric functions to estimate relationships between the response and explanatory variables. The nonparametric functions are estimated from observed data using spline smoothing, i.e., linear functions of covariates in a GLM are replaced by nonparametric spline functions in a GAM (Hastie and Tibshirani 1986, Wood 2006). The structure of the best model selected with age-related covariates, whether it was a GLM or a GAM (see Model selection), was then used to further investigate temporal variation in BS as well as possible effects of individual heterogeneity on BS trajectories.

\section{Accounting for heterogeneity in individual quality}

Observed (marginal estimates of) age-specific variation in BS depends on whether or not the correlation between BS and survival probability is taken into account (Cam and Monnat 2000). Heterogeneous survival across individuals leads to changes in the composition of a sample population as "frail" individuals readily die (and thus exit the sample), leaving only the most "robust" individuals in a sample at advanced ages (Vaupel and Yashin 1985). As a result, populationlevel estimates of age-specific BS can reflect patterns resulting from "within-generation phenotypic selection" (Endler 1986), rather than genuine age-specific variation in BS experienced by individuals (Cam et al. 2002b, Naves 2007).

To account for population-level processes influencing age-specific variation in BS, we first implemented a fixed effect of life span into our best model (i.e., the best GLM or GAM with AFR and EXP effects). As was done for AFR and EXP, we compared categorical, continuous, and spline-transformed effects of life span on BS. Furthermore, we considered various interactive effects of life span, AFR, and EXP on age-specific BS. As outlined by van de Pol and Verhulst (2006), such an approach can explicitly account for heterogeneity in timing of appearance (i.e., variation in AFR) and disappearance (i.e., variation in life span) from the breeding population. We went one step further by also modeling variation in breeding history (i.e., variation in EXP).

Next, to (1) parsimoniously account for repeated measures on individuals, in which some may be of higher reproductive quality than others, and (2) account for variation in reproductive quality across individuals, we added an individual random effect (denoted as ID) to our best model that included life span and other agerelated covariates. The final model thus contained fixed effects of life span, possibly AFR and EXP, as well as an individual random effect, which is necessary to fully account for the effects of individual heterogeneity on estimates of breeding performance (BS in our case; see Cam et al. 2002b, van de Pol and Verhulst 2006).
Temporal change in breeding success

We could not consider a model that simultaneously accounted for both an individual random effect and a random effect of "year" because asymptotic convergence of parameter estimates could not be achieved (despite the use of a supercomputer). However, we did include simultaneously a fixed effect of year (i.e., treated either continuously or as a factor in order to control for temporal change in BS) and an individual random effect to control for environmentally driven changes in BS in our best performing model, while controlling for heterogeneity in individual quality.

Separately, we also examined the influence of temporal variation in BS (e.g., resulting from climatic conditions, predation events, food shortages, and so forth) on age-specific BS using a random effect of Year. We modeled Year using a random effect because we had no motive to suspect a specific pattern for the influence of year on BS (e.g., a systematic trend). The random Year effect was implemented into the best-performing model with age-related covariates. All random effects were fit using the "mgcv" package in R 2.6.0 ("gamm" procedure).

\section{Model selection}

Because AFR, EXP, and life span are all linked to age sensus stricto, we calculated a variance inflation factor (i.e., package "car," procedure "vip" in R version 2.6.0; R Development Core Team 2007) to address the possible issue of multicolinearity prior to model selection (Neter et al. 1996).

To evaluate our predictions laid out in the introduction, we first conducted comparisons of GLMs containing the same biological covariate but parameterized in different ways (e.g., models with AFR or EXP parameterized as continuous linear, quadratic, or cubic covariates, or parameterized as categorical covariates). Throughout, Akaike's Information Criterion, AIC (Akaike 1973) and Akaike model weights $\left(w_{i}\right)$ were used to compare models and determine which model(s) served as the best approximation(s) to the data. For each estimated slope parameter $\left(\beta_{i}\right)$ that appeared in the best approximating model(s), we assessed the precision of each $\beta_{i}$ based on the extent to which $95 \%$ confidence intervals for each $\beta_{i}$ overlapped zero (Graybill and Iyer 1994).

After determining the best parameterization for each covariate, we then used AIC to compare GLMs with additive or interactive effects of covariates with different biological meaning (e.g., AFR, EXP). Using the covariates retained in the best GLM, and the nature of the covariate effects (i.e., additive or interactive, linear, quadratic or cubic, and so forth), we then built a less constrained GAM. For example, if the best GLM involved an interaction between a linear effect of AFR and a quadratic effect of EXP, the corresponding GAM would contain an interaction between EXP and AFR; however, the parametric forms would be replaced with a spline function to relax the previous linear or polyno- 
mial constraints. The degree of smoothness in modeled spline relationships between BS and explanatory covariates was estimated as part of the GAM procedure. If the GAM outperformed the best GLM, all further modeling was done using GAMs.

Next, we used the previous best-performing model (GLM or GAM) to model the effects of individual heterogeneity on age-specific BS. In this endeavor, we considered alternative parameterizations of the life span covariate (e.g., linear, quadratic, categorical, and so forth) as well as additive and interactive effects of life span with AFR and EXP on age-specific BS. For reasons laid out before, individual random effects were also included in models designed to account for individual heterogeneity (see Accounting for heterogeneity in individual quality).

Earlier stages of model selection suggested that GAMs did a better job of explaining our BS data than GLMs. Therefore, random effects were implemented by using generalized additive mixed models (i.e., "GAMMs," as opposed to "GLMMs"). When examining GAMMs, one cannot compare them to GLMs or GAMs using AIC because of its reliance on maximization of full likelihoods (Wood 2006). In such cases, we evaluated the statistical utility of a GAMM, and its effectiveness in accounting for age-specific variation in BS arising from individual heterogeneity, by using graphical diagnostics, assessing the precision of estimated parameters with $95 \%$ confidence intervals, and evaluating appropriate test statistics (i.e., we used a plural approach to making inference from models whenever information-criterion methods could not be used; Scheiner 2004). We also compared GAMMs to each other in some cases using AICs (e.g., GAMMs containing different parameterization for temporal change in BS; e.g., fixed vs. continuous).

\section{RESUlts}

\section{Age-related changes in breeding success}

All tests for multicolinearity in models containing AFR, EXP, and life span yielded estimates of variance inflation factors $<7.07$. Because all variance inflation factors were $<10$, our models did not present any serious issues concerning multicolinearity (Neter et al. 1996). Given these results, we considered models with additive and interactive effect of age-related covariates.

Among GLMs, our top-performing model (Table 1, second model from top) indicated that BS changed over life as a function of AFR (treated as a categorical factor), a cubic effect of EXP, and their interaction. Even though many of the coefficients for the AFR and (cubic effect of) EXP interaction were imprecisely estimated (95\% CIs overlapped 0), ignoring them led to a considerable increase in AIC (Table $1 ; \Delta_{i}=81$ ). The cubic effect of EXP in the best-performing GLM implied that either (1) the marginal estimate of BS, after an increase early in life and a decrease at intermediate ages, did indeed improve again late in life (Fig. 1), or (2) the

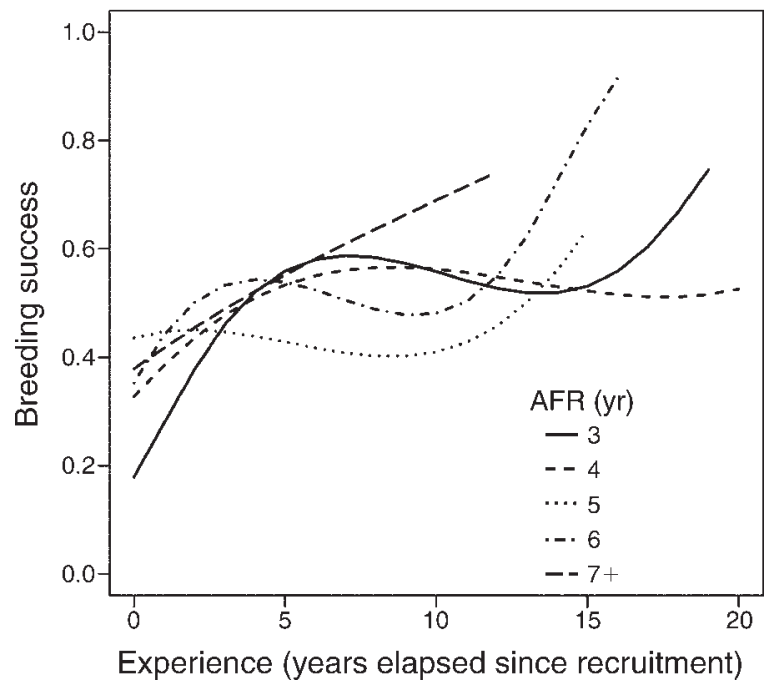

FIG. 1. Trajectories of age-specific breeding success (BS) in relation to age at first reproduction (AFR) and breeding experience in the Black-legged Kittiwake (Rissa trudactyla). Estimates were obtained from the best-performing generalized linear model (GLM) before potential sources of individual and temporal heterogeneity were accounted for (see Table 1, second model).

cubic polynomial "forced" a bimodal pattern between BS and EXP, which might not be an accurate depiction of the underlying relationship between EXP and BS at the population level, but only the closest fit to the data among the set of models examined.

To examine the validity of the parametric constraints in the best GLM, we compared it to an alternative GAM with "unconstrained" effects of age-related covariates on BS. When added to the set of candidate GLMs, the GAM was a superior fit to the data relative to the bestperforming GLM described previously (top model in Table $1 ; \Delta_{i}=6 ; \beta_{\mathrm{AFR}}=-0.17,95 \%$ CI: -0.16 to -0.18 ; spline effect of EXP: $\chi^{2}=100.60$, estimated df (edf) $=$ 4.23, $P<0.001$; spline effect of EXP and AFR interaction: $\chi^{2}=220.90$, edf $=13.26, P<0.001$ ). For the sake of conciseness, we did not include the figure associated with the GAM. However, this model not only was a better fit to the data, but also showed that for individuals surviving up to advanced ages, improvement in BS was observed in most recruitment groups. Interestingly, only early recruitment groups (i.e., AFR = 3 or 4 years) exhibited another decline in BS at very advanced ages ( $>20$ years). Yet, only a small sample of individuals existed at advanced ages (Appendix A). Based on these results, we considered the best-performing model (i.e., a GAM) in further analyses of BS as a function of both observed (i.e., AFR, EXP, life span) and unobserved heterogeneity (i.e., random effect of "ID").

\section{Accounting for heterogeneity in individual quality}

When a fixed effect of life span was added (last model in Table 2) to the GAM described previously (first 
TABLE 2. Selection results for models testing the effects of age at first reproduction and experience on age-specific breeding success, while accounting for heterogeneity in survival chances (life span effects).

\begin{tabular}{|c|c|c|c|c|c|}
\hline Model & AIC & edf & $\Delta_{i}$ & $\exp \left(-0.5 \Delta_{i}\right)$ & $w_{i}$ \\
\hline $\mathrm{S}(\mathrm{AFRc} \times \mathrm{EXP})+\mathrm{S}(\mathrm{EXP} \times$ life $\mathrm{span})$ & 10913.29 & 24.65 & 0 & 1 & 0.49 \\
\hline $\mathrm{S}(\mathrm{AFRc} \times \mathrm{EXP})+\mathrm{S}(\mathrm{EXP} \times$ life span $)+\mathrm{S}(\mathrm{AFRc} \times$ life span $)$ & 10913.4 & 24.67 & 0.11 & 0.95 & 0.46 \\
\hline $\mathrm{S}(\mathrm{AFRc} \times \mathrm{EXP})+\mathrm{S}($ life span $)$ & 10919.01 & 20.52 & 5.72 & 0.06 & 0.03 \\
\hline $\mathrm{S}(\mathrm{AFRc} \times \mathrm{EXP})+\mathrm{S}(\mathrm{AFRc} \times$ life span $)$ & 10920.08 & 21.97 & 6.79 & 0.03 & 0.02 \\
\hline $\mathrm{S}(\mathrm{AFRc} \times \mathrm{EXP})+$ life span & 10936.71 & 19.11 & 23.42 & 0 & 0 \\
\hline
\end{tabular}

Note: All models considered are GAMs; abbreviations are as in Table 1.

model in Table 1), AIC improved by 33.69 units. Because life span (by controlling for the selective disappearance of individuals within the breeding population) led to improvement in model fit, we further considered alternative parameterizations of life span. The top model including life span accounted for $49 \%$ of the model weight, and the second-best model accounted for $46 \%$ (Table 2). In the top model, all interactions were significant (spline effect of AFR and EXP interaction: edf $=16.75, \chi^{2}=151.90, P<0.001$; spline effect of EXP and life span interaction: edf $=6.90, \chi^{2}=74.78, P<$ 0.001 , which was also the case for the second-bestperforming model (spline effect of AFR and EXP interaction: edf $=16.75, \chi^{2}=151.87, P<0.001$; spline effect of EXP and life span interaction: edf $=6.89, \chi^{2}=$ 74.80, $P<0.001$; spline effect of AFR and life span interaction: edf $=0.03, \chi^{2}=9.35, P=0.002$ ).

To account for (unobserved) heterogeneity in individual quality, we considered an individual random effect and added it to the previously defined best GAM (i.e., generalized additive model). Such a model, a GAMM (i.e., generalized additive mixed model), could not be fit if one of the covariates was included in a splinetransformed interaction more than once (e.g., AFR involved twice in: $\mathrm{S}(\mathrm{AFR} \times \mathrm{EXP})+\mathrm{S}(\mathrm{AFR} \times$ life span $)$ + random effect(ID), where $S$ stands for a spline transformation; see Wood 2006). However, a model with an individual random effect and a three-way splinetransformed interaction between AFR, EXP, and life span converged successfully. The triple interaction term was statistically significant (spline effect of AFR, EXP, and life span interaction: edf $=9$, estimated rank $=9, F=$ 29.19, $P<0.001$ ), and random variation in BS across individuals was detected (individual random effect centered at zero: $\mathrm{SD}=0.93$, residuals $=0.87$ ).

Of great importance, this GAMM accounted for all demographic sources of individual heterogeneity in agespecific BS (i.e., selective appearance, selective disappearance, variation in breeding history, correlative structure across repeated measures, and random variation across individuals). According to this model, improvement in BS at advanced ages disappeared across all recruitment groups, and the pattern observed in agespecific BS surfaces was unimodal for all recruitment groups (Fig. 2), with no detectable late-life improvement in BS. Maximal BS levels decreased as individuals delayed recruitment: BS exceeded 0.6 for individuals recruiting at age $3(\mathrm{BS}=0.66,95 \% \mathrm{CI}: 0.45-0.87)$ and age 4 years $(\mathrm{BS}=0.63,95 \% \mathrm{CI}$ : $0.50-0.76)$, followed by individuals recruiting at age $5(0.58,95 \%$ CI: $0.47-0.69)$, $6(\mathrm{BS}=0.53,95 \% \mathrm{CI}: 0.40-0.66)$, and $7+$ years $(0.47$, 95\% CI: 0.29-0.65).

Based on this model, we calculated senescence rates for each recruitment group by calculating the slope between the highest $\mathrm{BS}$ and the lowest BS values for each BS surface (Fig. 2). We found that recruits of 4 years old experienced the highest senescence rate (i.e., $-0.034)$, followed by recruits of 3,6 , and 5 years old (i.e., respectively, $-0.30,-0.029,-0.027)$. The lowest senescence rate was associated with $7+$ year-old recruits (i.e., -0.025 ).

The model also provided some insights on how BS surfaces shifted as recruitment was delayed, as experience was gained, and as life span increased (Fig. 3). Estimated values of BS reached a maximum at 10 years of experience, for a realized life span of 20 years old (Fig. 3, top right), followed by a dramatic decline in BS late in life (Fig. 3, top left). Recruitment at age 3 was associated with a slightly higher maximal BS, given that an individual lived up to 15 years old (Fig. 3, center). The confidence bounds associated with each BS surface confirm the unimodal shape of BS surfaces across recruitment groups (Fig. 3).

\section{Temporal changes in breeding success}

A GAMM accounting for both individual effects and temporal variation in BS (i.e., individual and temporal random effect) failed to converge. Simultaneous estimation of multiple random effects in GAMMs is known to be computationally intensive (Wood 2006).

We first examined temporal variation in BS (i.e., random effect of YEAR) using a GAMM with a splinetransformed triple interaction of AFR, EXP, and life span. The interaction term was statistically significant (spline effect of AFR, EXP, and life span interaction: edf $=9$, estimated rank $=9, F=41.24, P<0.001)$ and the temporal random effect was nonnegligible (temporal random effect centered at zero: $\mathrm{SD}=0.34$, residuals $=$ 0.99). Most of the temporal variation in BS took place early in life, but decreased with AFR, and as individual gained experience (Appendix B).

We also considered two similar GAMM (i.e., with a spline-transformed triple interaction between AFR, EXP, and life span), accounting for heterogeneity in 

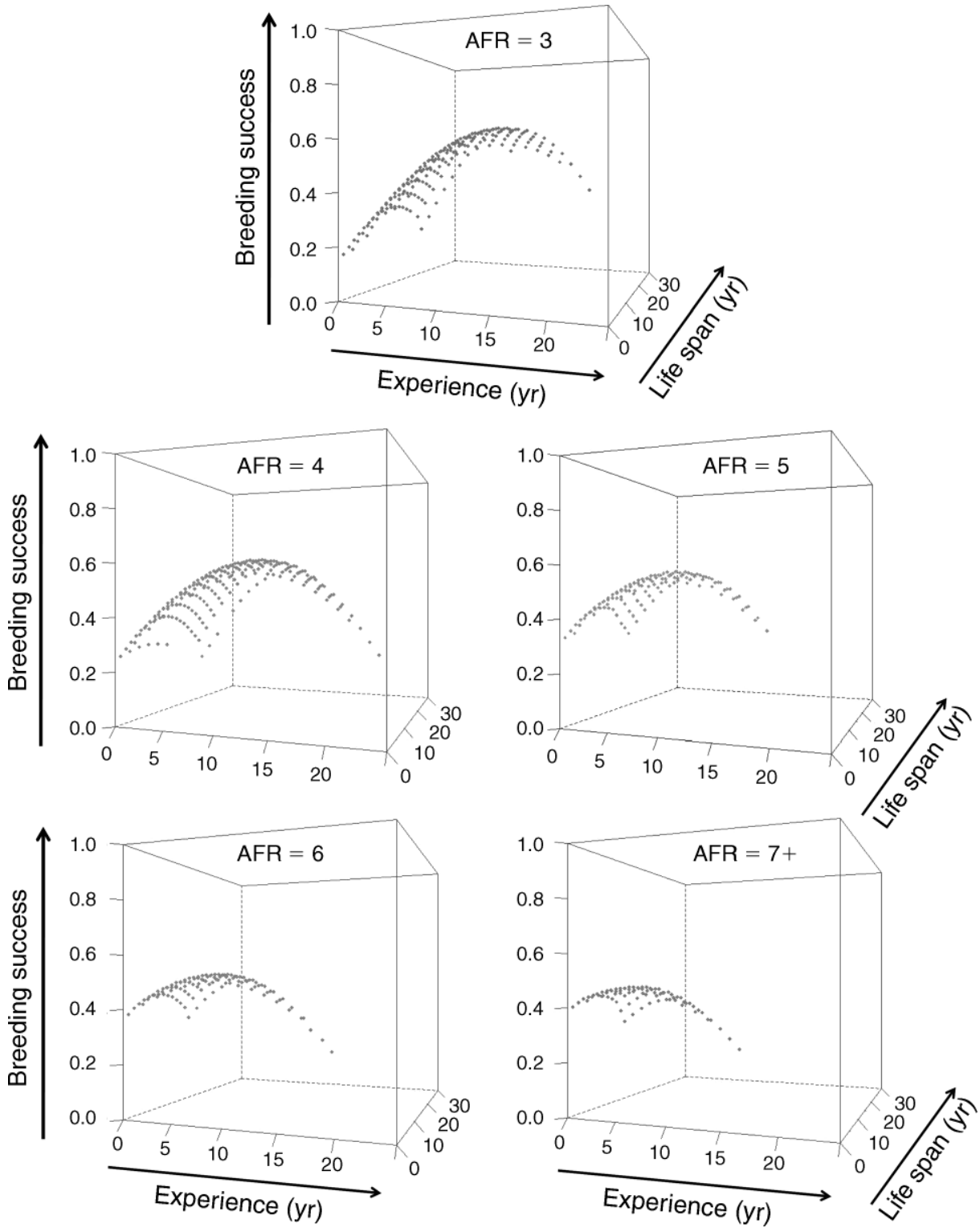

FIG. 2. Breeding success (BS) surfaces across recruitment groups: age at first reproduction (AFR, in years) as a function of experience (EXP, the time in years elapsed since first reproduction) and life span (years), once all potential sources of heterogeneity were accounted for. Individual heterogeneity is accounted for by an individual random effect, "ID." Estimates were obtained from the following GAMM: BS $\sim$ spline $($ AFR $\times$ EXP $\times$ life span $)+$ random effect ID.

individual quality (i.e., random effect of ID), and controlling for temporal change in BS by adding a fixed effect of YEAR either treated as a continuous covariate or as a factor. Models controlling for both individual differences in BS (i.e., random effect of ID) and for environmental stochasticity (i.e., random effect of YEAR), did converge successfully. However, in a GAMM considering a fixed effect of YEAR, the estimated effect was not significant and the confidence bounds overlapped zero $\left(\mathrm{YEAR}=3.6 \times 10^{-3}, 95 \% \mathrm{CI}\right.$ : $-1.1 \times 10^{-3}$ to $8.3 \times 10^{-3}, P=0.442$ ). As AIC is comparable across GAMM models, adding a fixed effect of YEAR did not improve model fit $(\mathrm{AIC}=36530.82$ for the GAMM without a fixed effect of YEAR, vs. AIC = 36531.03 for the GAMM with the fixed effect of YEAR). A GAMM with YEAR as a factor did not improve model fit either $($ AIC $=36$ 949.05). Moreover, none of the factorial levels were significant, and all estimates overlapped 0 . Therefore, it seems that most of the unobserved heterogeneity is a reflection of heterogeneity in individual quality rather than temporal change in BS.

\section{Discussion}

In this study, we addressed which recruitment tactics (i.e., early vs. delayed recruitment) yield the highest BS 

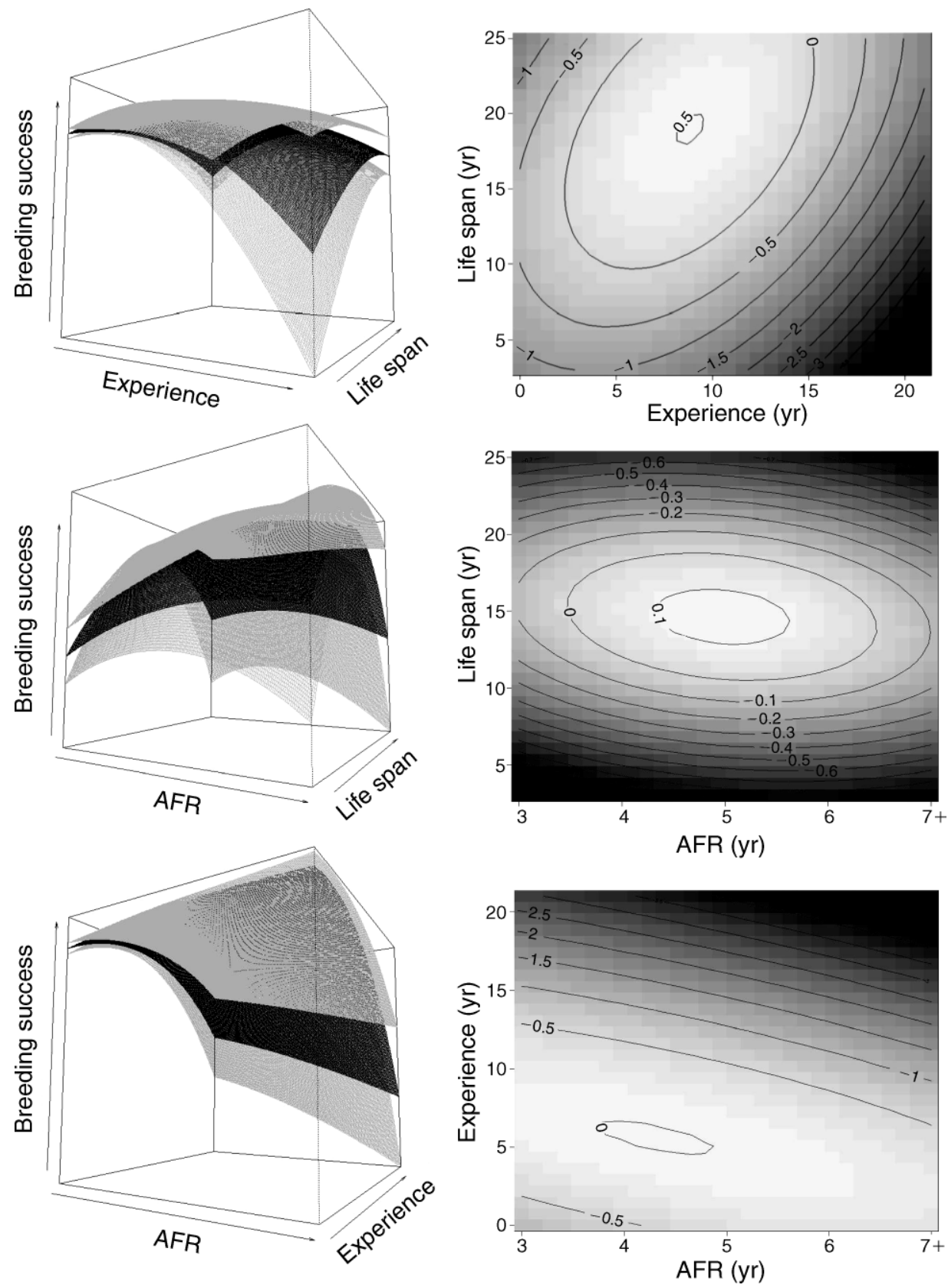

FIG. 3. Depictions of the influence of paired covariates (AFR and EXP, AFR and life span, EXP and life span) on breeding success (BS). Estimates were obtained from the GAMM defined in Fig. 2. The 95\% confidence bounds (gray surfaces) associated with each BS surface (black) are based on spline-transformed estimates. The contour plots serve as an alternative to threedimensional plots, display BS peaks more accurately, and show how they change as a function of age-related covariates.

across ages, and whether recruitment tactics led to contrasting reproductive senescence profiles. We knew from past work (Aubry et al. 2009) that pre-breeders reaching age 4 years had the highest probability of recruiting in the following year if they survived (i.e., reached age 5), and also experienced the highest BS in the year of recruitment. However, fitness prospects and life history consequences of individual decisions in terms of recruitment age are more completely understood by studying variation in BS throughout life; BS in the first breeding attempt is only a snapshot of the age-specific reproductive profile. We addressed this while accounting for heterogeneity in individual quality, a key component of the apparent influence of age on demographic parameters (e.g., Cam et al. 2002a, van de Pol and Verhulst 2006).

In accordance with hypothesis (3), we consistently found that early recruits (i.e., 3 years old) experienced 
low BS at recruitment, and thus started their reproductive life with a handicap (Figs. 1 and 2), perhaps as a consequence of a lack of "pre-breeding experience." We also found that individuals delaying recruitment exhibited higher BS in the recruitment year than did early recruits (Figs. 1 and 2). These results, consistent throughout all analyses (i.e., GLM, GAM, GAMM), confirm that pre-recruitment experience matters in determining levels of BS early in the reproductive career of individuals. Such pre-recruitment experience is likely to allow for an increase in skills related to reproduction (e.g., mate coordination, foraging abilities, nest construction, and so forth) through prospecting. It has been shown in the kittiwake that most individuals "prospect" for higher quality sites (e.g., Danchin et al. 1991, 1998, Cadiou et al. 1994). Before reproduction begins, prospectors gather local information based on the performance of their conspecifics, which permits selection of more productive habitats in the following year(s), probably to ensure high levels of BS in the recruitment year (e.g., Boulinier and Danchin 1997). Individuals that delayed recruitment gave up early-life breeding opportunities and achieved high BS at first reproduction. Potentially, they might have queued for high-quality sites and obtained a favorable breeding ground necessary to achieve such high levels of BS at recruitment. To test this, one would have to simultaneously examine the relationship between habitat selection, individual quality, and age-specific BS.

Following recruitment, improvements in BS were observed for all individuals that gained breeding experience, including early recruits (Figs. 1 and 2), suggesting that individuals can catch up from early-life deficits and perform increasingly better throughout life. The most rapid increase in BS was observed in early recruits, as their initial $\mathrm{BS}$ at recruitment year was so low, there was scope for substantial improvement later in life. On the other hand, individuals that delayed recruitment experienced a less impressive improvement because they initially experienced high levels of BS, leaving little room for enhancement.

Following the initial increase in BS across all recruitment groups, the different set of analyses provided different results. Under a GLM (Fig. 1) modeling framework, we did not detect senescence in BS in any of the recruitment groups, and an improvement in BS was even observed late in life (Fig. 1). We improved model fit by considering unconstrained relationships between the selected covariates and BS (Table 1; see GAM); this highlights the utility of spline transformations in obtaining realistic age-specific trajectories of demographic parameters such as BS.

These models, however, did not account for individual heterogeneity, and all showed late-life improvement in BS (in all recruitment groups under the best GLM in Fig. 1, and in most recruitment groups under the GAM; figure not presented here for the sake of conciseness). Recruitment-level increases in BS may only represent a handful of "robust" individuals that survive to advanced ages, and might not accurately reflect variation in BS within individuals, if individuals within recruitment groups do not die at the same rate (i.e., heterogeneity in individual quality can bias group-level estimates of BS, survival, and so forth; Vaupel and Yashin 1985). Even though the inclusion of life span in our best GAM did improve model fit (Tables 1 and 2), we were concerned that defining frailty a priori with "life span" might not fully distinguish between "robust" and "frail" individuals. Such an approach might only take into account "within-cohort phenotypic selection" (Ender 1986), but not other unobserved components of heterogeneity among individuals (e.g., Lewis et al. 2006). Therefore, we used an approach explicitly accounting for variation in reproductive quality across individuals (i.e., individual random effect), and for variability in recruitment age, life span, and breeding experience. According to this approach, there was a noticeable difference in the observed peak in BS across recruitment groups (Figs. 2 and 3), which occurred earlier in individuals delaying recruitment. However, across all recruitment groups, maximal BS levels were attained by individuals reaching intermediate experience levels. Interestingly, the balance between pre- and postrecruitment experience differed across recruitment groups, but resulted in similar timing of maximal BS (i.e., age 10-13 years, sensu stricto). Assuming that individuals were physiologically mature at age 2, maximum BS was attained at high levels of postbreeding experience (i.e., 10 years of experience) in early recruits (i.e., AFR $=3$ ), and at high levels of prerecruitment experience (i.e., 5 years of pre-recruitment experience) in individuals delaying recruitment (i.e., AFR $=7+$ ). Individuals recruiting at intermediate ages (i.e., AFR $=5$ ) demonstrated balanced pre- and postrecruitment experience ( 2 and 3 years, respectively) when BS reached its maximal value. As a follow-up, it would be worth testing which "recruitment tactic" (e.g., cumulating pre-recruitment, or post-recruitment experience) is associated with the highest fitness payoff (Maynard Smith 1982), BS being only a component of fitness. It would also be worth testing whether such tactics relate to differences in phenotypic quality. This would allow us to assess if low-quality individuals, suspected to delay recruitment, improve their BS by cumulating pre-recruitment experience, and if higher quality individuals (i.e. early recruits) increase BS by gaining breeding skills post-recruitment.

When we focused on the second half of BS trajectories, individuals delaying recruitment showed slightly lower senescence rates (i.e., lowest senescence rate observed in $7+$ year-old recruits) than "early recruits" (i.e., highest senescence rates observed in 4-year-old recruits). In accordance with the cumulative cost hypothesis (1), early recruits showed strong senescence decline in BS at advanced ages, when heterogeneity was properly accounted for. Individuals 


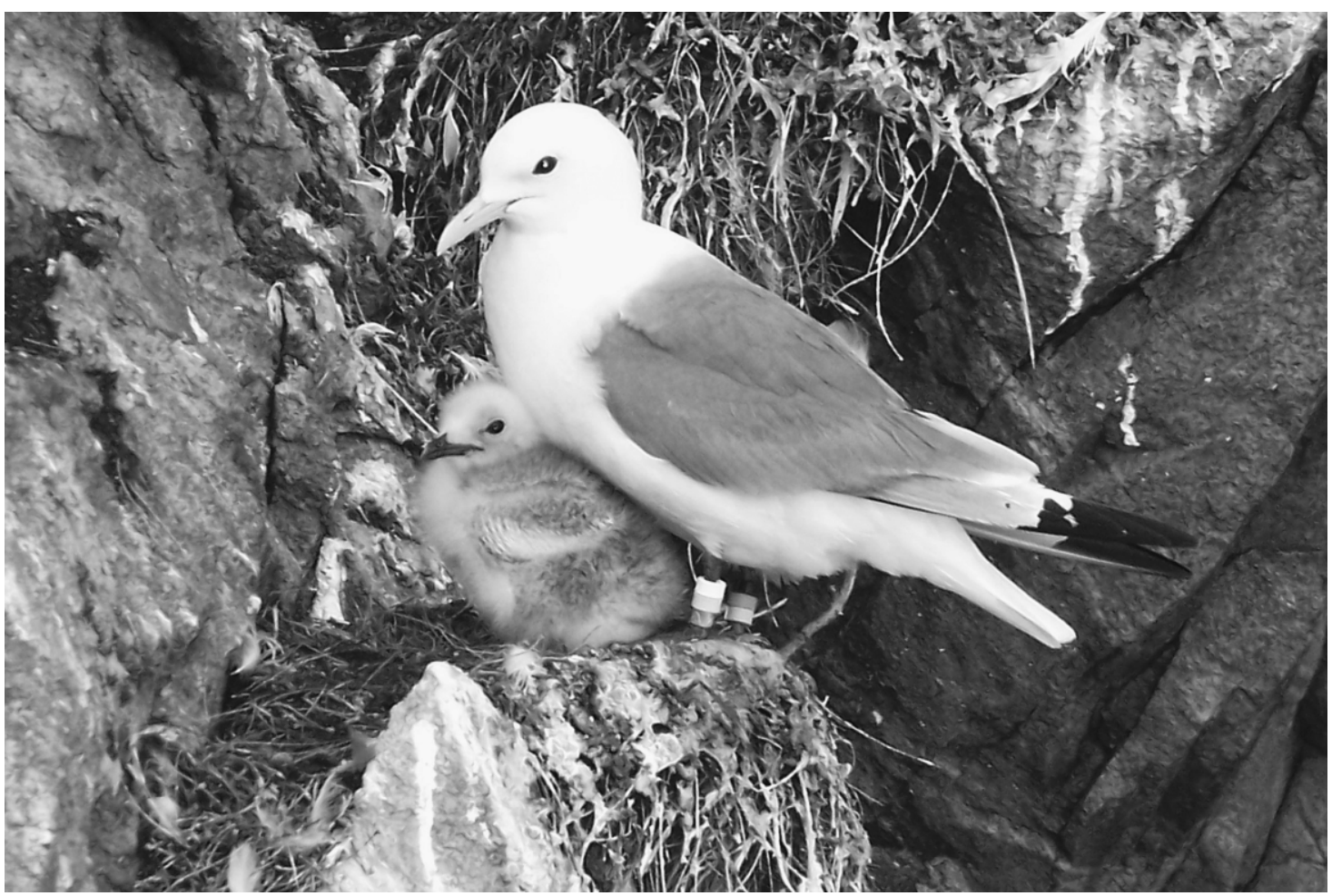

PLATE 1. "WJNOP" (banding combination: white yellow black orange pistachio) is a female kittiwake born in 1999 in colony 3. She went on a pelagic foray and was found hanging out with another 3-year-old in colony 4 in 2002 (but the two being shy, nothing happened). In 2003, she settled in 5O115 (colony 5, cliff O, nest-site 115) with a new partner and had two chicks. For a few years she produced chicks with uneven success and switched partners several times. In 2007, she finally met her match (JOOBR, a 3-year-old male), and the two have been seen successfully breeding on $5 \mathrm{O} 25$ since then. Photo credit: L. M. Aubry.

delaying recruitment, on the other hand, showed weaker senescence in BS. The idea of delayed costs of reproduction that could be expressed later in life, in terms of reproductive senescence, actuarial senescence, or both, has only emerged recently in empirical studies of long-lived species, even though it has been a central idea in senescence theory for more than 70 years. If costs of reproduction are not demographically evident in the short term, payment could reveal itself later in life, even at advanced ages. For example, an "invest now, pay later" reproductive tactic could culminate in delayed or cumulative costs of reproduction (e.g., Orell and Belda 2002), and could translate into late-life senescence in both reproduction and survival. We still need to address whether these trade-offs also underlie senescence in survival. Such delayed trade-offs should be of special interest to evolutionary ecologists, because they have the potential to clarify the simultaneous evolution of reproductive strategies and senescence.

BS also varied across years, but annual effects primarily affected individuals early in life (Appendix B), suggesting that experienced individuals were able to buffer out the effects of temporal variation in environmental conditions on BS, which can substantially improve fitness in stochastic environments (Lewontin and Cohen 1969, Tuljapurkar 1982). Thus, in addition to bet-hedging (e.g., Slatkin 1974), iteroparity (e.g., Orzack and Tuljapurkar 1989), and longevity (e.g., Metcalf and Koons 2007), a stochastically changing environment may also select for the devotion of resources to acquiring reproductive experience. Temporal change, however, only contributed to subtle changes in BS early in life.

In accordance with Cam et al. (2002b), our work suggests that most of the changes in BS across ages in the population were accounted for by an interaction between age-related covariates (i.e., observed heterogeneity), and an individual random effect (i.e., unobserved heterogeneity). Heterogeneity in survival (e.g., frailty models) was first looked at in human demography starting some twenty years ago (Vaupel and Yashin 1985). However, developments and applications regarding heterogeneity in demographic rates such as BS in wild animal population are fairly recent (e.g., Cam et al 2002b, Barbraud and Weimerskirch 2005, Fox et al. 2006, Van de Pol and Verhulst 2006, Royle 2008). We found that in addition to unobserved heterogeneity, one should also consider interactions between observable age-related covariates to account for the multiplicity of life-course events that define individual differences in BS. The triple interaction between recruitment age, experience, and life span seemed to account for the 
selective appearance and disappearance of individuals, and for the diversity of possible "breeding lives" (i.e., number of breeding attempts in the life course).

Even though a flurry of scientific papers have been studying trade-offs between a variety of traits and latelife reproduction (e.g., Bérubé et al. 1999, Reid et al. 2003, van de Pol and Verhulst 2006, Reed et al. 2008), only a handful used proxies for differences in individual quality (e.g., life span). To our knowledge, Balbontín et al. (2007), along with Reed et al. (2008), are the only ones relating senescence in BS to age-related traits while accounting for heterogeneity in individual quality by using mixed models. However, we went one step further by using models (i.e., GAMMs) accounting for both random effects controlling for heterogeneity in individual quality, and splines to examine unconstrained relationship between BS and age-related covariates (see Ezard et al. [2007] for similar models applied to age, phenology, and individual fitness relationships). By doing so, we explicitly show how to obtain individually based estimates of BS by simultaneously accounting for the fact that individuals recruit, breed (successfully or not), and die at various ages, without constraining the relation between BS an age-related covariates (i.e., smooth splines).

We next aim to explicitly measure the relative contributions of observed and unobserved heterogeneity to age-specific trajectories of survival in the kittiwake, and then develop appropriate models for integrating all lifecycle parameters and measuring forces of natural selection on life history decisions (e.g., Coulson et al. 2006).

\section{ACKNOWLEDGMENTS}

We thank the Conseil Général du Finistère for facilitating our work in the nature reserve of Goulien Cap Sizun (Brittany, France), managed by the Société pour l'Étude et la Protection de la Nature. We express our deep gratitude to all the people who contributed to data collection, and especially to Jacques Nisser (Office National de la Chasse et de la Faune Sauvage) for his precious help. We are most grateful to Evan Cooch for access to his LINUX server at Cornell University. Help from Florent Bled, Carlo Giovanni Camarda, Cornelia Krauss, Jessica Metcalf, Samuel Pavard, David Thomson, and James Vaupel greatly improved our work. In particular, we thank Anne Charmantier for her valuable comments on the manuscript. L. M. Aubry was funded by the Max Planck Institute for Demographic Research in Germany.

\section{Literature Cited}

Agresti, A. 1990. Categorical data analysis. John Wiley, New York, New York, USA.

Akaike, H. 1973. Information theory and an extension of the maximum likelihood principle. Pages $267-281$ in B. N. Petran and F. Csaki, editors. International symposium on information theory. Second edition. Akadéniai Kiadi, Budapest, Hungary.

Aubry, L. M., E. Cam, and J.-Y. Monnat. 2009. Habitat selection, age-specific recruitment, and reproductive success in a long-lived seabird, the black-legged kittiwake. Pages 285-292 in D. L. Thomson, E. G. Cooch, and M. J. Conroy, editors. Modeling demographic processes in marked popula- tions series. Environmental and ecological statistics. Volume 3. Springer, New York, New York, USA.

Balbontín, J., I. G. Hermosell, A. Marzal, M. Reviriego, F. de Lope, and A. P. Møller. 2007. Age-related change in breeding performance in early life is associated with an increase in competence in the migratory barn swallow (Hirundo rustica). Journal of Animal Ecology 76:915-925.

Barbraud, C., and H. Weimerskirch. 2005. Environmental conditions and breeding experience affect costs of reproduction in Blue Petrels. Ecology 863:682-692.

Bérubé, M., M. Festa-Bianchet, and J. T. Jorgenson. 1999. Individual differences, longevity, and reproductive senescence in bighorn ewes. Ecology 80:2555-2565.

Boulinier, T., and E. Danchin. 1997. The use of conspecific reproductive success for breeding patch selection in territorial migratory species. Evolutionary Ecology 11:505-517.

Cadiou, B., J.-Y. Monnat, and E. Danchin. 1994. Prospecting in the kittiwake, Rissa tridactyla: different behavioural patterns and the role of squatting in recruitment. Animal Behaviour 47:847-856.

Cam, E. 1997. Reproduction intermittente et corrélations phénotypiques entre composantes de la fitness chez la mouette tridactyle Rissa tridactyla. Thèse de l'Université Paris VI, France.

Cam, E., B. Cadiou, J. E. Hines, and J.-Y. Monnat. $2002 a$. Influence of behavioural tactics on recruitment and reproductive trajectory in the kittiwake. Journal of Applied Statistics 291:163-185.

Cam, E., J. E. Hines, J.-Y. Monnat, J. D. Nichols, and E. Danchin. 1998. Are adult nonbreeders prudent parents? The kittiwake model. Ecology 79:2917-2930.

Cam, E., W. A. Link, E. G. Cooch, J.-Y. Monnat, and E. Danchin. 2002b. Individual covariation between life-history traits: seeing the trees despite the forest. American Naturalist 159:96-105.

Cam, E., and J.-Y. Monnat. 2000. Apparent inferiority in first time breeders in the kittiwake: the role of heterogeneity among age-classes. Journal of Animal Ecology 69:380-394.

Charmantier, A., C. Perrins, R. H. McCleery, and B. C. Sheldon. 2006. Quantitative genetics of age at reproduction in wild swans: Support for antagonistic pleiotropy models of senescence. Proceedings of the National Academy of Sciences (USA) 103:6587-6592.

Charnov, E. L. 1997. Trade-off-invariant rules for evolutionarily stable life histories. Nature 387:393-394.

Coulson, J. C., and J. A. Fairweather. 2001. Reduced reproductive performance prior to death in the Black-legged Kittiwake: senescence or terminal illness? Journal of Avian Biology 32(2): 146-152.

Coulson, T., T. G. Benton, P. Lundberg, S. R. X. Dall, B. E. Kendall, and J.-M. Gaillard. 2006. Estimating individual contributions to population growth: evolutionary fitness in ecological time. Proceedings of the Royal Society B 273:547555 .

Danchin, E., T. Boulinier, and M. Massot. 1998. Conspecific reproductive success and breeding habitat selection: implications for the study of coloniality. Ecology 79:2415-2428.

Danchin, E., B. Cadiou, J.-Y. Monnat, and R. Rodriguez Estrella. 1991. Recruitment in long-lived birds: conceptual framework and behavioural mechanisms. University of Ottawa Press, Ottawa, Canada.

Endler, J. A. 1986. Natural selection in the wild. Princeton University Press, Princeton, New Jersey, USA.

Ezard, T. H. G., P. H. Becker, and T. Coulson. 2007. Correlations between age, phenotype, and individual contribution to population growth in common terns. Ecology 88: $2496-2504$.

Fox, G. A., B. E. Kendall, J. W. Fitzpatrick, and G. E. Woolfenden. 2006. Consequences of heterogeneity in survival probability in a population of Florida scrub-jays. Journal of Animal Ecology 754:921-927. 
Graybill, F. A., and H. K. Iyer. 1994. Regression analysis: concepts and applications. Duxbury Press, Belmont, California, USA.

Harshman, L. G., and A. J. Zera. 2007. The cost of reproduction: the devil in the details. Trends in Ecology and Evolution 222:80-86.

Hastie, T., and R. Tibshirani. 1986. Generalized additive models. Statistical Science 13:297-318.

Lack, D. 1968. Bird migration and natural selection. Oikos 191: $1-8$.

Lewis, S., S. Wanless, D. A. Elston, M. D. Schultz, E. Macket, M. Du Toit, J. G. Underhill, and M. P. Harris. 2006. Determinant of quality in a long-lived colonial species. Journal of Animal Ecology 75:1304-1312.

Lewontin, R. C., and D. Cohen. 1969. On population growth in a randomly varying environment. Proceedings of the National Academy of Sciences (USA) 62:1056-1060.

Maynard Smith, J. 1982. Evolution and the theory of games. Cambridge University Press, Cambridge, UK.

Metcalf, C. J. E., and D. N. Koons. 2007. Environmental uncertainty, autocorrelation and the evolution of survival. Proceedings of the Royal Society B 274:2153-2160.

Naves, L. C., E. Cam, and J.-Y. Monnat. 2007. Pair duration, breeding success and divorce in a long-lived seabird: benefits of mate familiarity? Animal Behaviour 733:433-444.

Neter, J., M. H. Kutner, C. J. Nachtsheim, and W. Wasserman. 1996. Applied linear statistical models. Fourth edition. Richard D. Irwin, Homewood, Illinois, USA.

Nevoux, M., H. Weimerskirch, and C. Barbraud. 2007. Environmental variation and experience-related differences in the demography of the long-lived black-browed albatross. Journal of Animal Ecology 76:159-167.

Nol, E., and J. N. M. Smith. 1987. Effects of age and breeding experience on seasonal reproductive success in the song sparrow. Journal of Animal Ecology 561:301-313.

Nur, N. 1988. The cost of reproduction in birds: an examination of the evidence. Ardea 76:155-168.

Nussey, D. H., L. E. B. Kruuk, A. Morris, and T. H. CluttonBrock. 2007. Environmental conditions in early life influence ageing rates in a wild population of red deer. Current Biology 17:1000-1001.

Orell, M., and E. J. Belda. 2002. Delayed cost of reproduction and senescence in the willow tit Parus montanus. Journal of Animal Ecology 711:55-64.

Orzack, S. H., and S. Tuljapurkar. 1989. Population dynamics in variable environments. VII. The demography and evolution of iteroparity. American Naturalist 133:901-923.
Partridge, L., and N. H. Barton. 1983. Evolution of aging: testing the theory using Drosophila. Genetica 91:89-98.

R Development Core Team. 2007. R. version 2.6.0. R: a language and environment for statistical computing. $\mathrm{R}$ Foundation for Statistical Computing, Vienna, Austria.

Reed, T. E., L. E. B. Kruuk, S. Wanless, M. Frederiksen, E. J. A. Cunningham, and M. Harris. 2008. Reproductive senescence in a long-lived seabird: rates of decline in late life performance are associated with varying costs of early reproduction. American Naturalist 171:89-101.

Reid, J. M., E. M. Bignal, D. I. McCracken, and P. Monaghan. 2003. Age-specific reproductive performance in red-billed choughs Pyrrhocorax pyrrhocorax: patterns and processes in a natural population. Journal of Animal Ecology 72:765-776.

Reznick, D. N., M. J. Bryant, D. Roff, and C. K. Ghalambor. 2004. Effect of extrinsic mortality on the evolution of senescence in guppies. Nature 431:1095-1099.

Ricklefs, R. E., and A. Scheuerlein. 2001. Comparison of agingrelated mortality among birds and mammals. Experimental Gerontology 36:845-857.

Royle, J. A. 2008. Modeling individual effects in the CormackJolly-Seber model: a state-space formulation. Biometrics 64(2):364-370.

Scheiner, S. M. 2004. Experiments, observations, and other kinds of evidence. Pages 51-72 in M. L. Taper and S. R. Lele, editors. The nature of scientific evidence: statistical, philosophical, and empirical considerations. University of Chicago Press, Chicago, Illinois, USA.

Slatkin, M. 1974. Hedging one's evolutionary bets. Nature 250: 704-705.

Stearns, S. C. 1992. The evolution of life histories. Oxford University Press, Oxford, UK.

Tuljapurkar, S. D. 1982. Population dynamics in variable environments. II. Correlated environments, sensitivity analysis and dynamics. Theoretical Population Biology 21: $114-140$.

van de Pol, M., and S. Verhulst. 2006. Age-dependent traits: a new statistical model to separate within- and betweenindividual effects. American Naturalist 167:764-771.

van Noordwijk, A. J., and G. de Jong. 1986. Acquisition and allocation of resources: their influence on variation in life history tactics. American Naturalist 128:137-142.

Vaupel, J. W., and A. I. Yashin. 1985. Heterogeneity's ruses: Some surprising effects of selection on population dynamics. American Statistician 39:176-185.

Wood, S. N. 2006. Generalized additive models: an introduction with R. Chapman and Hall, CRC Press, Boca Raton, Florida, USA.

\section{APPENDIX A}

Study population, data collection, and sample specifications (Ecological Archives E090-175-A1).

\section{APPENDIX B}

Temporal changes in breeding success (Ecological Archives E090-175-A2). 